\title{
Ni régimen de prima media ni régimen de ahorro individual: mejor los dos*
}

\section{Neither Premium Pension Scheme nor Individual Savings Scheme: Better both}

\section{\{ Stefano Farné $\}^{\star \star}$}

Recibido: I de diciembre de 20I9. Aprobado: I de junio de 2020. Dor: https://doi. org/I0.1860I/25390406.n5.03

Magíster en Economía de la Universidad de Londres, Reino Unido. Director del Observatorio del Mercado de Trabajo y la Seguridad Social, Universidad Externado de Colombia. 


\section{Resumen}

Ya hace años el problema pensional colombiano está sobre la mesa del debate de los hacedores de política nacionales e internacionales. Su solución resulta ser compleja e involucra el abordaje de al menos cuatro tipos de problemáticas: sostenibilidad, equidad, cobertura y suficiencia. Este documento hace un resumen de las principales propuestas avanzadas para dar solución a los mencionados problemas y expone una propuesta elaborada por el Observatorio del Mercado de Trabajo y la Seguridad Social que se fundamenta en un sistema de pilares complementarios — de prima media, el primero, y de ahorro individual, el segundo-y se caracteriza por no introducir cambios demasiados radicales en las reglas de funcionamiento del sistema pensional actualmente vigente en el país.

\section{Palabras clave}

Sistema pensional, prima media, ahorro individual, reforma pensional.

\section{Abstract}

For years the Colombian pension problem has been on the table of debate by national and international policymakers. Its solution turns out to be complex and involves addressing at least four types of issues: sustainability, equity, coverage, and sufficiency. This document summarizes the main advanced proposals to solve the problems mentioned above. It sets out a proposal prepared by the Labor Market and Social Security Observatory based on a complementary pillars system -with the first average premium and individual savings. The second second-and is characterized by not introducing too radical changes in the operating rules of the pension system currently in force in the country.

\section{KeYwords}

Pension system, premium pension scheme, individual savings scheme, pension reform.

\section{INTRODUCCIÓN}

Desde hace años el problema pensional colombiano está sobre la mesa del debate de los hacedores de política nacional e internacional. Su solución resulta ser compleja e involucra el planteamiento de al menos cuatro tipos de problemáticas: sostenibilidad, equidad, cobertura y suficiencia. 
La sostenibilidad financiera afecta al Régimen de Prima Media (RPM). En efecto, a causa de la desactualización de sus parámetros de operación se generan cuantiosos subsidios en favor de todos los afiliados que logran pensionarse. Estos subsidios no son financieramente cubiertos con recursos endógenos al régimen, sino que demandan la intervención del erario público. Sin embargo, en un futuro, cuando el competidor Régimen de Ahorro Individual (RAIS) llegue a su maduración, es muy probable que la cobertura estatal por deslizamiento de la pensión mínima ${ }^{\mathrm{I}}$ y por garantía de pensión mínima requiera de considerables contribuciones por parte del presupuesto nacional para garantizar las prestaciones económicas del aumentado número de derecho habientes a una pensión.

De diferentes formas y con intensidad diversa, la equidad afecta tanto el RPM como al RaIs. No obstante, lo que más llama la atención son los cuantiosos subsidios que otorga el RPM y que crecen en valor con el aumentar de los ingresos de los pensionados, alcanzando cifras millonarias.

Ambos regímenes exhiben una baja cobertura tanto en la fase activa (porcentaje de afiliados/cotizantes) como en la pasiva (porcentaje de pensionados).

Finalmente, la suficiencia de la mesada pensional —es decir, el valor de la mesada que debería garantizar un nivel de bienestar a los pensionados no muy diferente del que habían alcanzado antes de la jubilación- se ha vuelto una cuestión crítica del rais, esto como consecuencia de la caída que ha experimentado en los últimos años la tasa de rendimiento reconocida por los fondos de ahorro pensional gestionados por las Administradoras de Fondos de Pensiones (AFP) y de los constantes progresos en materia de mortalidad experimentados en el país.

Superar estos cuatro obstáculos manteniendo la actual estructura pensional dual, en la cual los afiliados pueden escoger entre los antagónicos subsistemas de capitalización individual y de prima media, no aparenta ser la mejor opción de política.

Tampoco parecen optimas las soluciones extremas de marchitar el RPM o de dejar a las Afp la administración de un solo componente voluntario.

Más bien, los dos subsistemas deberían ser integrados en un sistema mixto con componentes de capitalización y al mismo tiempo de prima media,

I En el 20I8, el pago por parte de la nación de una fracción del incremento anual de la pensión mínima (fenómeno denominado deslizamiento salarial o pensional, dado que la segunda debe siempre ser igual al primero) fue de 26675 millones de pesos. En el 20I9, se han presupuestado 3 856 millones y se espera que esta cifra aumente con los años, en la medida en que el salario (pensión) mínimo aumenta por encima de la inflación y la productividad de la economía. 
que permitiría aprovechar el hecho de que los dos regímenes están expuestos a riesgos de diferente naturaleza en parte no correlatos entre ellos.

En este sentido, el presente documento presenta una propuesta de reforma pensional que se fundamenta en un sistema de pilares complementarios. Se trata de una opción que ya hace parte de la canasta de propuestas de reforma pensional avanzadas hasta ahora en el país. Nuestra contribución se caracteriza por tratar de evitar traumatismos propios de un cambio demasiado radical en las reglas de funcionamiento del sistema. Por ejemplo, no requiere de una nueva reforma tributaria y mantiene la estructura solidaria y de recaudo. Además, si bien algunas de las modificaciones sugeridas requieren de ulteriores estudios para ser cuantificadas en sus exactas dimensiones, se ha hecho un esfuerzo para ser concretos en materia de cambios propuestos.

El artículo se desarrolla de la siguiente forma: en la sección dos que sigue inmediatamente esta introducción se hace un resumen de las principales propuestas de reforma al sistema pensional colombiano desarrolladas hasta ahora. En la sección tres se expone nuestra propuesta y en una cuarta y última sección se destacan unas conclusiones y se prevén algunas acciones para el futuro.

\section{Algunas propuestas de reforma pensional}

\section{Fedesarrollo}

Siguiendo el enfoque multipilar del Banco $\mathrm{Mundial}^{2}$, la propuesta de Fedesarro$1 \mathrm{l}^{3}$ se fundamenta en un esquema de pilares compuesto de cuatro elementos. Primero, Colombia Mayor se consolidaría como un pilar cero no contributivo, con una función puramente asistencialista, focalizado en otorgar subsidios a la población vulnerable. Segundo, el pilar uno consistiría en un esquema de reparto administrado por el Estado, que recibirá las contribuciones correspondientes al primer salario mínimo de todos los trabajadores cotizantes. El pilar dos, gestionado por las AFP, administrará los aportes obligatorios de los trabajadores por la parte de ingresos superiores a un salario mínimo bajo un esquema de capitalización individual. Finalmente, un pilar tres se encargaría de administrar el ahorro voluntario previsional de los trabajadores.

2 Banco Mundial, Envejecimiento sin crisis. Políticas para la protección de los ancianos y la promoción del crecimiento (Washington: Oxford University Press, 1994).

3 Leonardo Villar y David Forero. Elementos para una propuesta de reforma del sistema de protección económica para la vejez en Colombia, Cuadernos Fedesarrollo 58 (Bogotá: Fedesarrollo, 2018). 
En cuanto a las prestaciones económicas reconocidas, el afiliado que cumple con los requisitos establecidos para pensionarse tendrá derecho a una mesada igual a un salario mínimo legal vigente del pilar uno y a una renta vitalicia del pilar dos cuyo valor dependerá del monto ahorrado durante su vida activa. Se elimina, así, el esquema de retiro programado del régimen obligatorio dejándolo como una opción solo para el régimen de pensiones voluntarias.

El elemento que caracteriza la propuesta de Fedesarrollo es la reducción progresiva del subsidio a la pensión de salario mínimo otorgada por el pilar uno, subsidio que persiste aun con los incrementos en la edad de jubilación y las cotizaciones mensuales que se proponen introducir. Para esto, al momento del reconocimiento de la pensión, un porcentaje del capital ahorrado por los trabajadores en el pilar dos (hasta un $25 \%$ ) se transferirá al pilar uno con el objetivo de cubrir parte del capital faltante estimado para financiar una mesada de salario mínimo. Dado que el monto absoluto de la transferencia al pilar uno se incrementa con el ahorro acumulado en el pilar dos, a partir de cierto nivel — aquel que financiaría una pensión de entre 2,5 y 3 salarios mínimos (5, según estimaciones más recientes) — los subsidios desaparecen. En la práctica, para trabajadores con niveles de ahorro mayores a este umbral, la transferencia desde el pilar dos equivaldría de manera exacta al monto absoluto del subsidio estimado por la prestación otorgada en el pilar uno. En estos casos, el sistema termina funcionando como un esquema de ahorro individual sin subsidios, haciendo la claridad que los rendimientos asumidos para la acumulación en el pilar uno no tienen por qué ser iguales a los efectivamente recibidos en el pilar dos.

De acuerdo con esta propuesta casi todas las pensiones verían reducido el subsidio recibido 4 , no solo las más altas.

Ahora bien, aunque Villar y Forero afirman que "para evitar otorgar subsidios a los trabajadores de altos ingresos, la presente propuesta plantea un gradiente de transición, que reduce paulatinamente el subsidio otorgado por el Pilar Uno a medida que aumenta la reserva actuarial de ahorro del individuo"5, en realidad los subsidios son función solamente del ahorro pensional obligatorio derivado de una actividad laboral formal y no del nivel de ingreso de los beneficiarios.

Además, un punto crítico del funcionamiento del sistema propuesto por Fedesarrollo es el cálculo del ahorro de cada participante como primer paso para

4 La variación del monto de los subsidios, sin embargo, depende de forma importante de los parámetros de la simulación utilizados (especialmente de la tasa de rendimiento, de la fecha de ingreso al sistema y la densidad de cotización).

5 Villar y Forero, Elementos para una propuesta de reforma, I5. 
la determinación del subsidio al cual se tendrá derecho. A pesar de que el pilar uno es, como afirman los autores, un sistema de reparto, es decir un sistema donde solo existe un fondo común, no resulta claro cómo se determinaría el ahorro pensional de cada individuo. Para ello sería necesario tener registro de los aportes a las cuentas individuales y a la tasa de rentabilidad, al no estar en un sistema de mercado debería ser fijada de forma exógena.

Relacionado con lo anterior, otro elemento merece atención. Un componente central de la propuesta de Fedesarrollo es el cálculo del subsidio máximo que recibiría un trabajador que a lo largo de su vida activa haya cotizado sobre un salario mínimo, al cumplir 65 años de edad y 1 I50 semanas de cotización. Este valor será la referencia para estimar los subsidios de todos los demás trabajadores. Aquí sobresalen dos puntos. Primero, dado que la estimación de este subsidio máximo está sujeta a los supuestos usados en la simulación actuarial, cualquier modificación que se realice sobre estos - principalmente sobre la tasa de rentabilidad y la densidad de cotización- implicaría un cambio en el monto del subsidio imputable a todos los trabajadores. En segunda instancia, el sistema podría estar entregando subsidios distintos a personas con iguales niveles de ingreso, pero trayectorias ocupacionales diferentes. Por ejemplo, un individuo que cotice de manera continua durante i 50 semanas, al final de su ciclo laboral, tendrá un subsidio totalmente distinto a uno que cotice durante el mismo número de semanas y el mismo salario, pero que haya experimentado entradas y salidas del mercado laboral formal a lo largo de su vida.

Nótese que bajo el modelo propuesto, la pensión contributiva de los trabajadores equivaldrá a la suma del beneficio obtenido en el pilar uno equivalente siempre a una mesada de un salario mínimo-y la renta vitalicia derivada de los ahorros restantes en el pilar dos, luego de deducir la transferencia al pilar uno mencionada anteriormente. Por tanto, dependiendo de los supuestos usados en el cálculo del subsidio máximo, el capital trasladado desde el pilar uno cambia y, en consecuencia, también su pensión total (suma de los beneficios otorgados en el pilar uno y dos).

Además, la propuesta de Fedesarrollo incluye cambios en los mecanismos de fijación de las comisiones de administración y los seguros previsionales. En el primer caso se propone un modelo de comisión en función de los activos administrados y la rentabilidad obtenida. Para los seguros de invalidez y sobrevivencia se plantea un mecanismo de subasta como el existente en países como Chile o Perú.

Asimismo, se proponen algunos mecanismos para integrar el sistema de beneficios económicos periódicos (вер), como un pilar intermedio entre el pilar cero (no contributivo) y el pilar uno de reparto simple. Entre otros se considera: impedir cualquier retiro de los ahorros en BEP que no sean para 
financiar rentas vitalicias, y que se debe hacer obligatoria la transferencia a BEP de todo el ahorro contributivo cuando no se alcance una pensión, acumulado ya sea en el primer o segundo pilar. Además, se proponen casos específicos de relaciones laborales en los cuales la cotización a BEP debe ser obligatoria, por ejemplo, contratos laborales con una temporalidad menor a un mes o de tiempo parcial, o empresarios informales o cuenta propia que coticen sobre sus ingresos brutos (monotributo). Adicionalmente, se recomienda incrementar el subsidio desde el actual $20 \%$ de los aportes efectuados en las cuentas BEP por el afiliado a un $50 \%$ y la fijación de un aporte inicial por parte del Estado - inicialmente de tres millones de pesos- que se materializa solo en la liquidación de la renta vitalicia.

Respecto al programa de Colombia Mayor se mencionan tres elementos principales. Primero, un ajuste en las edades de acceso de tal manera que siempre sean superiores a la edad estatutaria de pensión. Segundo, aumentar la cobertura del programa pasando del Sisbén como elemento de focalización hacia un criterio de riqueza o renta. Tercero, incrementar el monto del subsidio, actualmente de entre 40000 y 75000 pesos mensuales, acercándolo al valor de la línea de indigencia ( I 7605 pesos mensuales en el 2018). Esto representaría un costo inmediato del o,2 \% del producto interno bruto (РІв) que se financiaría con impuestos de renta a las pensiones altas.

En términos de ajustes paramétricos se plantea:

- Un aumento gradual en la edad de pensión hacia los 65 años tanto para hombres como para mujeres. Para estas últimas se mencionan algunos mecanismos compensatorios como la reducción de las semanas a cotizar dependiendo del número de hijos

- Reducción de las pensiones de sobrevivencia desde el actual roo \% a un $75 \%$.

- Equiparación de las semanas mínimas de cotización necesarias para pensionarse a las I 50 exigidas actualmente en el rais.

- Eliminación de los aportes solidarios (del Fondo de Garantía de Pensión Mínima y del Fondo de Solidaridad Pensional).

- Incremento de la cotización en dos puntos porcentuales.

Cabe mencionar que la propuesta considera que el faltante derivado de la eliminación de los aportes solidarios al fsp deberá ser asumido por el presupuesto nacional, así como el aumento en los subsidios a los Bep. Según Fedesarrollo, este déficit será eventualmente cubierto con los excedentes generados por la reducción y eliminación de los subsidios a todas las pensiones. Sin embargo, al menos durante el tiempo que dure la transición, estos beneficios no se materializarían, lo que implica una carga adicional sobre presupuesto. 
Además, el incremento de los dos puntos porcentuales en la cotización afectaría fundamentalmente a los trabajadores de bajos ingresos. En efecto, para aquellos con ingresos altos la eliminación de los aportes solidarios compensaría este incremento. Por ejemplo, mientras que los trabajadores que cotizan sobre 25 salarios mínimos seguirán pagando el mismo i $8 \%$ de su cotización, los trabajadores con ingresos menores a cuatro salarios mínimos efectivamente verán incrementada su cotización en dos puntos porcentuales. La propuesta no aclara quién asumiría los dos puntos porcentuales de cotización, si los empleados o los empleadores.

\section{Asociación Nacional de Instituciones Financieras (anif) ${ }^{6}$}

El eje central de la reforma estructural pensional de ANIF es el marchitamiento gradual del RPM y para esto se propone impedir la entrada de nuevos afiliados. En este caso, Colpensiones seguirá cumpliendo con sus obligaciones pensionales actuales, así como las que se generarían por los afiliados anteriores a la fecha de cierre.

La propuesta de ANIF considera los siguientes ajustes paramétricos en el RPM durante el tiempo que tarde el cierre definitivo:

- Reducción de la pensión mínima al 75 \% del salario mínimo.

- Reducción de la tasa de reemplazo en veinte puntos porcentuales para ingresos superiores a un salario mínimo.

- Incremento gradual de la edad de pensión en cinco años tanto para hombres como para mujeres.

- Reducción de la pensión sustitutiva hacia un 75 \% de la pensión del causante.

- Ampliación del periodo de tiempo para el cálculo del ibl a toda la vida laboral del trabajador.

Aunque los ajustes paramétricos mencionados previamente son explícitos para el RPM, la propuesta de ANIF menciona algunos cambios que involucran al RAIS. Por ejemplo, sugiere que para poder "crear" un mercado de rentas vitalicias y de aseguramiento previsional en Colombia se requiere de diversos ajustes y modificaciones a la regulación. Entre las posibles medidas se destacan:

I. Acotar la garantía de pensión mínima hacia el 50\%-75\% del salario mínimo.

6 La siguiente descripción está basada en el documento Elementos para una Reforma Estructural Pensional (REP) de ANIF. 
2. Permitir "recalcular" las rentas vitalicias (como ocurre en Chile), manteniendo inalterada la obligación de la aseguradora.

3. Crear un mercado más profundo en materia de aseguramiento previsional.

4. Extender los tiempos de redención de los tes a treinta años, respecto de los vigentes diez-quince años, para así lograr un adecuado calce financiero a la hora de expedir rentas vitalicias?.

5. Trabajar en la línea de mayor certeza jurídica sobre beneficiarios de estos seguros, acotando las "sorpresas" de nuevos beneficiarios".

Claramente la posibilidad de que la mesada pensional sea inferior al salario mínimo exige una reforma constitucional paralela a las demás modificaciones paramétricas.

Adicionalmente, la propuesta considera la flexibilización y ampliación del Fondo de Garantía de Pensión Mínima (FGPM) a todos los afiliados del nuevo sistema eliminando la actual condición de ingresos o rentas superiores al salario mínimo.

ANIF, en vista de los altos costos políticos que implicarían todos estos ajustes, plantea una propuesta de reforma alternativa menos ambiciosa fiscalmente. Los puntos más sobresalientes de este nuevo proyecto son: se descartaría la idea de marchitar Colpensiones y los ajustes paramétricos en el RPM serían más moderados particularmente en dos casos: la garantía de pensión mínima se mantendría en el Ioo \% del salario mínimo y la reducción de la tasa de remplazo no sería de veinte, sino de diez puntos porcentuales. Los demás ajustes de la reforma inicial se mantendrían?

\section{Asofondos}

Asofondos propone la creación de un sistema contributivo único basado en el ahorro y la capitalización individual, en que tanto administradoras públicas como privadas compitan bajo las mismas condiciones ${ }^{\mathrm{IO}}$. Este sistema único, al

7 La intención es que al ampliar la maduración de los tes se consolide un activo financiero que tenga la misma duración que el tiempo promedio por el cual se paga una renta vitalicia, esto permitiría un adecuado ajuste entre ambos productos.

8 Sergio Clavijo, Nelson Vera, Ekaterina Cuéllar y Andrea Ríos, Elementos para una Reforma Estructural Pensional (REP) (Bogotá: ANIF, 20I7), 5 I.

9 Clavijo, Vera, Cuéllar et al., Elementos para una Reforma, 6r.

Io Santiago Montenegro, “El camino es el ahorro. Reforma integral para la vejez”, en Cecilia López Montaño y María Claudia Holstine (eds.), La reforma pensional en Colombia. El debate pendiente. Lecciones de América Latina (Bogotá: Cisoe y Banco de la República, 2019), I28-I55. 
ser de capitalización individual, desaparecería todos los subsidios pensionales que se reconocen actualmente en el RPM. Solo quedarían subsidiadas las pensiones de aquellos trabajadores que accedan al FGPM, es decir, principalmente de aquellos trabajadores con ingresos de salario mínimo o poco superiores.

Vale la pena resaltar que el sistema único propuesto por Asofondos, al igual que la reforma estructural de ANIF, llevarían a una baja substancial de las tasas de reemplazo de las mesadas que actualmente reconoce Colpensiones a todos los demás trabajadores. Según estimaciones del modelo actuarial del Observatorio del Mercado de Trabajo y la Seguridad Social, para trabajadores con ingresos superiores a cuatro salarios mínimos, dichas tasas se reducirían a porcentajes apenas superiores al $30 \%$ de los ingresos de los últimos diez años de vida activa de los afiliados. Para aquellos trabajadores con ingresos inferiores la tasa de reemplazo tiende a ser más alta, debido a la restricción constitucional que establece que ninguna pensión puede ser inferior al salario mínimo mensual vigente.

En general, el sistema pensional estaría dividido en tres esquemas. Primero, Colombia Mayor como programa asistencial donde todos los adultos mayores de 65 años que pertenezcan a Sisbén i y in recibirían un subsidio equivalente a la línea de pobreza. Como fuentes de financiación se propone incrementar los aportes que hoy en día hacen los trabajadores al FSP y recursos del Presupuesto General de la Nación. Como segunda instancia, un pilar semicontributivo enfocado en los trabajadores que no cumplen con los requisitos de capital o semanas para pensionarse. En este caso, las devoluciones de saldos se transformarían automáticamente en una renta vitalicia bajo el esquema de BEPS. $\mathrm{Y}$, finalmente, un esquema contributivo que seguirá la misma estructura del RaIs actual. Se propone la creación del Fondo de Reserva Pensional (Frp) que, al recibir los recursos del FGPM, asumiría también sus actuales funciones. Los aportes realizados al FRP variarían entre un I \% y un 2,5\% del IBC conforme con los ingresos del trabajador.

Por último, Asofondos propone que la administradora pública que funcione en el sistema tenga elementos de gobierno corporativo como los que posee actualmente el Banco de la Republica que garanticen independencia frente al Gobierno.

\section{Centrales obreras}

La propuesta de la Central Unitaria de Trabajadores de Colombia (CuT) y la Confederación de Trabajadores de Colombia (стс) aborda elementos tanto del 
esquema contributivo como no contributivo ${ }^{11}$. Respecto al primero, se formula eliminar la competencia actual entre regímenes estableciendo un único sistema de reparto público obligatorio, complementado con un esquema de ahorro voluntario administrado por las AFP. Esto implica que los activos gestionados actualmente por el RAIs pasarían al RPM administrado por Colpensiones.

Para las personas con contrato de prestación de servicios y otras figuras de contratación temporal, la propuesta considera que una vez finalizado el tiempo de trabajo, el Estado, mediante un mecanismo de aseguramiento, subsidie la cotización a salud y a pensiones de los empleados por máximo seis meses. Además, los trabajadores informales cotizarían el $4 \%$ de un salario mínimo y el restante i $2 \%$ lo aportaría el Estado, esto sin afectar los beneficios de la salud subsidiada o de la condición de beneficiario en el esquema contributivo.

Respecto al sistema no contributivo, la cUт y la стс plantean reemplazar Colombia Mayor por un programa de pensiones universales no inferior a medio salario mínimo, otorgada a todos los hombres mayores de 62 años y mujeres mayores de 57 años, más allá de las cotizaciones realizadas. Para financiar este programa se piensa principalmente:

a) Usar los recursos de Colombia Mayor, el PSAP y el FSP.

b) Impuestos a las pensiones altas.

c) Aportes voluntarios como se establece en BePs.

d) Destinar un dólar de cada barril exportado para consolidar un fondo que pague estas pensiones.

e) Hacer uso de las indemnizaciones sustitutivas y las devoluciones de saldos

Adicionalmente, mencionan algunos elementos para definir un régimen especial rural. Entre estos están: creación y formalización de asociaciones y organizaciones agrarias, como vínculo entre el Sistema General de Pensiones y los campesinos; un sistema flexible para el pago de cotizaciones que tome en consideración la estacionalidad de las cosechas y la alta volatilidad de los ingresos agrarios y finalmente sugieren que el aporte al sistema de pensiones que hagan los campesinos no debería implicar el pago completo al sistema de salud -que actualmente asciende al i 2,5\% del ingreso base de cotización de los trabajadores independientes - ni la perdida de los beneficios de la salud subsidiada. 


\section{Escuela Nacional Sindical (ens)}

La propuesta de las $\mathrm{ENS}^{\mathrm{I2}}$ se fundamenta en un sistema de tres pilares. El primer pilar, o piso de protección social, tiene un carácter universal y pretende asegurar un ingreso vitalicio antipobreza (medio salario mínimo) a todo adulto mayor más allá de sus contribuciones al sistema de pensiones. Estaría focalizado inicialmente en la población adulta mayor más vulnerable, con el objetivo de extenderlo progresivamente a toda la población mayor que hoy no recibe pensión. Se financiaría principalmente con

a) Impuestos.

b) Recursos del programa Colombia Mayor.

c) Aportes de las empresas que hoy se benefician del trabajo de los recicladores y de las ventas en kioscos, casetas, "puerta a puerta” y otras estrategias de externalización de ventas.

Además, la propuesta contempla el uso de las devoluciones de saldos e indemnizaciones sustitutivas de los afiliados que no cumplen requisitos y lo ahorrado en BEPs para aumentar ese ingreso mínimo.

El pilar dos funcionaria como el actual RPM, sin cambios, dado que no se consideran ajustes paramétricos. Este pilar es obligatorio para todos los trabajadores con ingresos inferiores a cuatro salarios mínimos. Finalmente, un tercer pilar para trabajadores que pueden cotizar con ingresos superiores a los cuatros salarios mínimos, que funcionaria a través de la apertura de cuentas de ahorro individual, de libre elección entre Colpensiones o en los fondos privados que, a su vez, le permitan un ingreso adicional y superior a la pensión que le asegura el segundo pilar.

La propuesta de la ens menciona dos puntos adicionales: primero, la garantía de pensión mínima para todos los trabajadores que hayan cumplido por lo menos con el $70 \%$ del tiempo de aportes mínimo exigido, para lo cual se les seguirá descontando la correspondiente cotización (supuestamente hasta completar el tiempo total no cotizado). Segundo, de conformidad con las recomendaciones del comité de libertad sindical de la orT, restablecer los derechos de negociación colectiva en materia pensional. 


\section{Hacia un sistema pensional de pilares}

En la misma línea que algunos de los modelos de reforma mencionados previamente $^{13}$ ), el sistema pensional propuesto se basa en un esquema de pilares que comprende tres tipos de pensiones:

I. Pensión plena contributiva: para los que completan un mínimo estatutario de años de cotizaciones y de edad.

2. Pensión parcial: también contributiva para los que no cumplen las condiciones para recibir una pensión plena, pero que tienen algún capital ahorrado, al cual se le van a sumar unos subsidios

3. Pensión asistencial: para los que no han nunca cotizado o que han cotizado, pero que sus niveles de ahorro/ingresos son muy bajos.

A cada tipo de pensión se asocia un arreglo institucional que corresponde, en el orden, a un sistema de pilares en el cual operarían Colpensiones y las AFP, al mecanismo de los Bep y al programa Colombia Mayor.

A continuación, se detalla el funcionamiento del sistema contributivo pleno y sucesivamente se avanzarán algunas consideraciones acerca de los BEP y Colombia Mayor en el entendimiento de que la operación de estos dos últimos programas deberá ser objeto de mayor estudio en un futuro.

En primera instancia, respecto a la situación vigente en la actualidad, al sistema contributivo se introducen los siguientes cambios paramétricos:

- Aumento de la edad de jubilación a 65 años, para hombres y mujeres ${ }^{\mathrm{I}}$.

- Aumento de las semanas de cotización a 1560, equivalentes a treinta años de contribuciones.

- Incremento en el IвC máximo de cotización a treinta salarios mínimos.

- Reducción de los cargos administrativos.

- Reducción de la pensión de sobrevivientes del primer pilar al $50 \%-75 \%$, siempre y cuando el beneficiario ya reciba una pensión del sistema público.

Y se contemplan nuevas figuras, tales como

- Créditos pensionales para mujeres.

- Compra de semanas.

- Compra del anticipo (etario) pensional.

- Entrada de nuevos actores como administradores de recursos (las Cajas de Compensación Familiar, por ejemplo).

I3 Orgulloso y Vásquez, "Retos de la política de protección social”, 2019; Villar y Forero, Elementos para una propuesta de reforma, 2018.

Estas edades aplicarían a todo tipo de pensión, contributiva plena y parcial y asistencial. 
El sistema contributivo se compone de dos pilares:

I. Un primer pilar de prima media, público y administrado por Colpensiones. Mensualmente recibe los recursos correspondientes al i6 \% de un IBC igual a un salario mínimo para todos cotizantes y a un I, $5 \%$ de todo iвc que excede el monto de un salario mínimo.

2. Un segundo pilar de ahorro individual administrado por las AfP. En la cuenta individual de cada afiliado se deposita toda contribución que exceda el aporte correspondiente al i6\% de un гвс igual a un salario mínimo ${ }^{\mathrm{I}}$.

\section{EL ESQUEMA SIN CAMBIOS EN LAS COMISIONES Y SEGUROS}

Si, por ejemplo, el trabajador gana un salario mínimo mensual, toda cotización ( 32500 pesos en el 20I9) irá al fondo común de Colpensiones (panel a de la figura I), que se distribuirá de la siguiente manera: un I3\% (I07655 pesos) iría al fondo común del primer pilar y el $3 \%$ (24 843) cubriría los seguros y comisiones. En el caso en que el trabajador recibiera bonificaciones con carácter salarial o efectuara trabajo extra, toda cotización que exceda el valor de 132500

\section{Figura 1.}

\section{Distribución de la cotización a Pensiones en el caso DE IBC DE 1 SALARIO MINIMO}

Panel (a): antes de las 1560 semanas

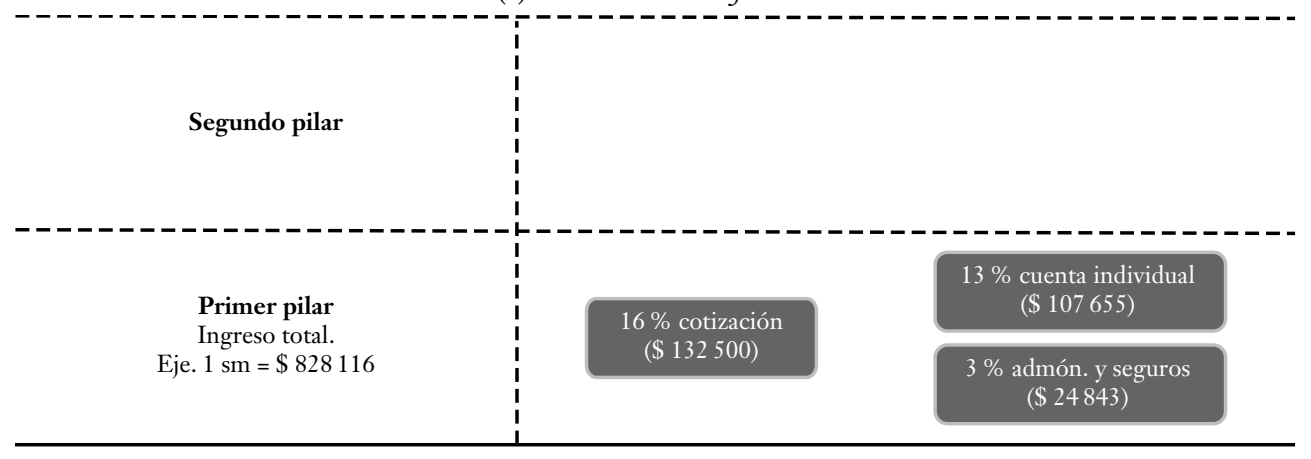

Aportes al FSP de los trabajadores con ingresos mayores a $4 \mathrm{sm}$. (Colombia Mayor)

\section{No aplica}

I5 Lo anterior implica que, respecto a las condiciones vigentes, desaparecería el Fondo de Garantía de Pensión Mínima - y la cotización correspondiente iría al fondo común de vejez de Colpensiones-y que todo aporte que exceda el i3 \% de un salario mínimo vigente — todo monto superior a 132500 pesos mensuales en el 2019 - iría depositado en la cuenta personal del afiliado, una vez descontadas las comisiones y los aportes solidarios. 
Panel (b): despues de las 1560 semanas

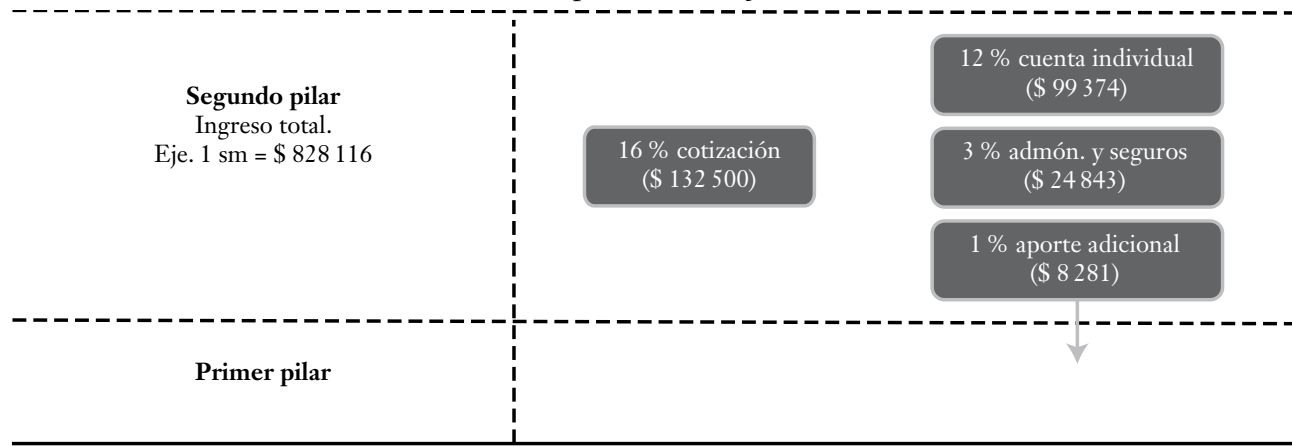

Aportes al FSP de los trabajadores con ingresos mayores a $4 \mathrm{sm}$. (Colombia Mayor)

Fuente: elaboración del autor.

pesos iría a una cuenta individual del afiliado, previo descuento de una comisión de administración y un aporte de solidaridad de i,5 \% el cual tendría como destino el fondo común de vejez del primer pilar. Una vez que el afiliado complete las 1560 semanas de cotización y no haya alcanzado la edad de retiro o habiéndola alcanzado decide continuar trabajado, este destinaría un punto porcentual de su cotización al primer pilar y el resto del valor de la cotización iría a su cuenta individual (descontadas las comisiones de administración; véase panel b de la figura r) del segundo pilar.

El caso de un trabajador con ingresos mensuales superiores al mínimo legal — por ejemplo, iguales a diez salarios mínimos — sería el siguiente (panel a figura 2). Hasta alcanzar las ${ }_{5} 60$ semanas cotizadas aportaría al primer pilar un $16 \%$ sobre el valor de un salario mínimo ( 132500 pesos), que se distribuirían de manera idéntica al caso de un trabajador que devengue solo un salario mínimo como el ejemplo del párrafo anterior. Adicionalmente, al primer pilar transferiría un I, 5 sobre los restantes nueve salarios mínimos de su IBC, equivalente a I I 796 pesos. Por otro lado, a su cuenta individual de capitalización del segundo pilar irá el II,5\% de nueve salarios mínimos (857 IOO pesos) y por comisiones pagaría un $3 \%$, equivalente a $22359 \mathrm{I}$ pesos. También pagaría un aporte de solidaridad de i \% sobre diez salarios mínimos con destino al Fondo de Solidaridad Pensional.

Una vez que el afiliado complete las 1560 semanas de cotización y no haya alcanzado la edad de retiro o habiéndola alcanzado decide continuar trabajado, destinaría un punto porcentual de su cotización al primer pilar y el resto del valor de la cotización iría a su cuenta individual (descontadas las 
comisiones de administración, véase panel b de la figura 2) del segundo pilar. Seguirá pagando el aporte solidario de r \% al Fondo de Solidaridad Pensional.

\section{FiguRA 2.}

Distribución de la cotización a Pensiones en el caso de ibC de diez salarios mínimos

Panel (a): antes de las 1560 semanas

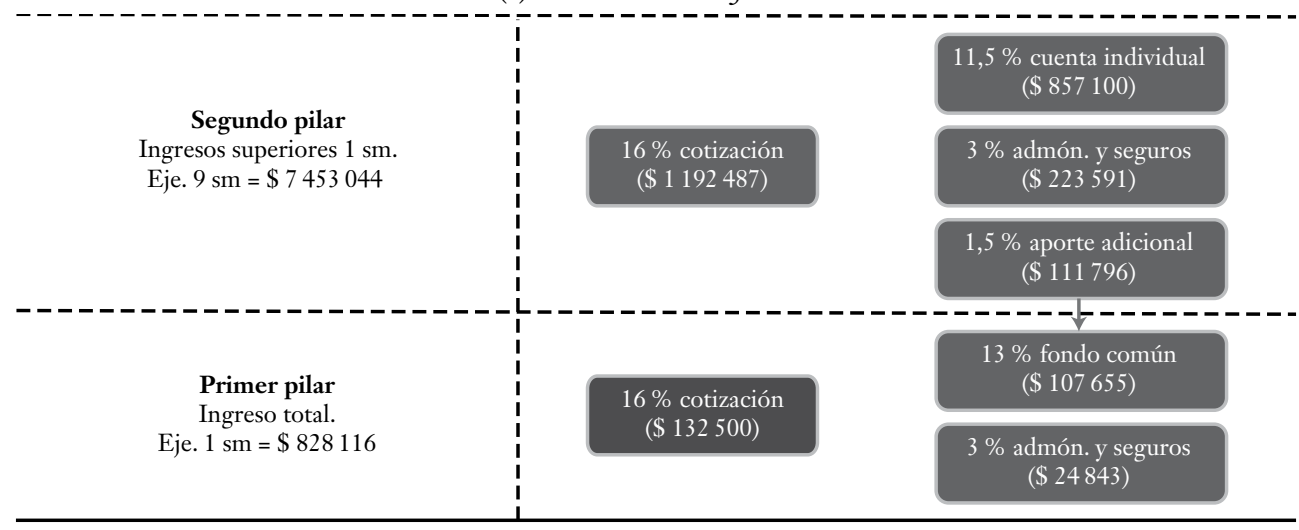

Aportes al FSP de los trabajadores con ingresos mayores a $4 \mathrm{sm}$. (Colombia Mayor)
$1 \%$ cotización de $10 \mathrm{sm}$ (\$ 82 812)

Panel (b): despues de las 1560 semanas

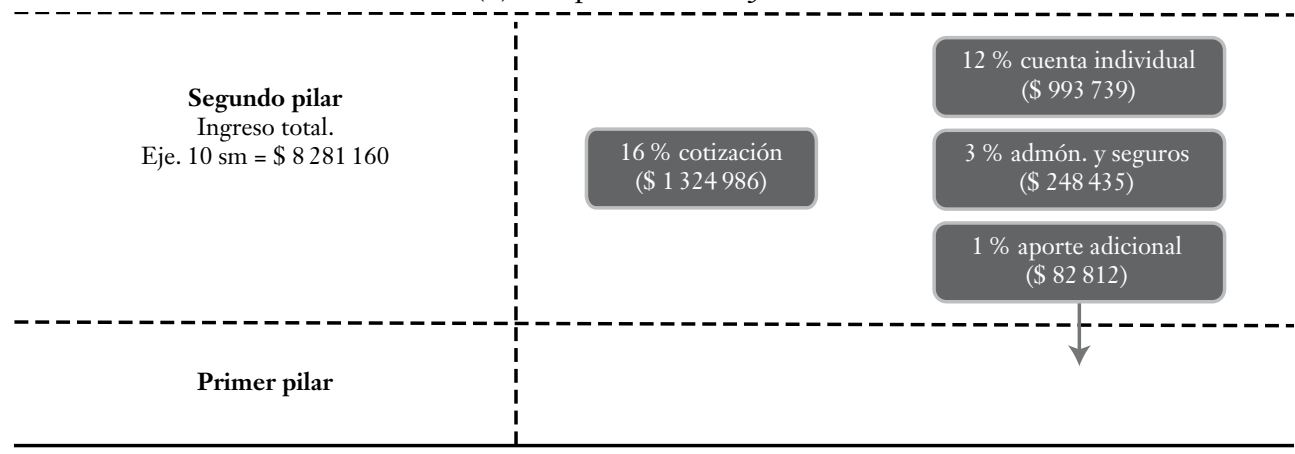

Aportes al FSP de los trabajadores con ingresos mayores a $4 \mathrm{sm}$. (Colombia Mayor)
$1 \%$ Cotización de $10 \mathrm{sm}$

(\$ 82 812)

Fuente: elaboración del autor.

Al cumplir los requisitos para pensionarse -65 años de edad y 30 años de cotizaciones - el afiliado tendrá derecho a una renta vitalicia por un valor constante en términos reales de un salario mínimo, pagadera por Colpensiones. Asimismo, lo acumulado en la cuenta individual del segundo pilar será destinado a incrementar esta pensión. De esta manera, el capital ahorrado se utilizará para acordar con la AFP un plan de retiro programado o para comprar 
una renta vitalicia con una compañía de seguro. En ambos casos, la mesada reconocida podrá ser inferior al salario mínimo vigente.

Ahora bien, puede darse el caso que el afiliado cumpla los 65 años de edad sin haber cotizado el número mínimo de semanas y no desee trabajar más. Con lo ahorrado en el segundo pilar o con sus ahorros personales podrá comprar el número de semanas que le faltan para completar lo exigido para pensionarse en el primer pilar. Y, viceversa, puede ser que el afiliado haya completado los 30 años de aportes y desee pensionarse antes de cumplir los $6_{5}$ años. En este caso podrá completar con sus ahorros lo que le falte actuarialmente para pensionarse en el primer pilar; es decir, podrá pensionarse pagando el anticipo de edad.

Finalmente, quien al cumplir los 65 años no tenga 1560 semanas cotizadas y tampoco tenga en su cuenta individual del segundo pilar el capital suficiente para comprar las semanas que le faltan, tendrá derecho a la indemnización sustitutiva por lo cotizado en el primer pilar y a la devolución de los saldos de sus ahorros en el segundo pilar y podrá integrarse al mecanismo de los BEP.

\section{El ESQUEMA CON CAMBIOS EN LAS COMISIONES Y SEgUROS}

Como se ha anticipado, se considera que hay margen para reducir los recargos administrativos que cobran Colpensiones en el primer pilar y las AFP en el segundo.

De hecho, Colpensiones que destina a su fondo de administración el I,०9\% de las cotizaciones recibidas, en el 2017 tuvo un excedente de 46554 millones de pesos que trasladó al fondo común destinado a financiar las pensiones de jubilación de todos sus afiliados. En el 20r8, el excedente fue de I 26476 millones. Según información de la Superintendencia Financiera, en el rais la sola comisión por concepto de administración de los aportes obligatorios sería más alta, de $\mathrm{r}, 25 \%$ en promedio. A pesar de ello, las AfP, a diferencia de Colpensiones, no contabilizan excedentes operacionales para distribuir entre sus clientes.

Farné y Nieto $^{16}$ han resaltado como el sistema de subasta adoptado en el régimen de capitalización chileno pudo reducir la comisión de administración por debajo del punto porcentual. Actualmente, la Afp chilena que menos cobra por administración descuenta apenas el o,77 \% del ingreso de cotización. Y al incluir las comisiones por concepto de seguros de invalidez y sobrevivientes la comisión total sube a $2,3 \%$, que representa un valor muy inferior al $3 \%$ que cobran Colpensiones y todas las AFP colombianas por disposición legal. 
Así que, los cargos administrativos que se cobran en Colombia podrían bajar. Su financiación podría ser como se propone a continuación.

Los seguros de invalidez y sobrevivientes se estipularán sobre el valor total del івс (pilar uno, más pilar dos) declarado por el aportante, serán financiados a través de un sistema de subasta y estarán a cargo de los empleadores. En Chile el esquema que aquí se propone ha dado lugar a primas de invalidez y sobrevivencia de I,4I \% sobre el IBC entre julio del 2016 y junio del 2018 y de $1,53 \%$ entre julio del 2018 y junio del 2020 . Con el exclusivo fin de simplificar nuestro argumento, suponemos que en Colombia el proceso de subasta de lugar a una prima de I, $5 \%$ sobre el IBC total. A parcial compensación por este nuevo recargo a la nómina se reduce en 0,5 puntos porcentuales el aporte total que actualmente hacen los empleadores al sistema pensional, de i $2 \%$ a I I, $5 \%$. El resultado final, entonces, sería un aumento de un punto porcentual tanto de la cotización pensional total, de $16 \%$ a $17 \%$ aproximadamente del ingreso de los afiliados, como de la sola cotización empresarial de I $2 \%$ a I3 \% ( I I, $5 \%$ más I, $5 \%$ por primas de seguros).

De forma similar, la comisión por administración de los fondos de inversión que se reconoce a las AFP podría ser fijada a través de un proceso de subasta. Y simultáneamente se podría autorizar la entrada de nuevos actores como las cajas de compensación familiar, por ejemplo, para incentivar ulteriormente la competencia entre administradoras. La comisión que así se establecería en el segundo pilar estaría completamente a cargo de los trabajadores y serviría de referencia para la operación del primer pilar.

Haciendo nuevamente alusión al caso de Chile, cabe resaltar cómo el proceso de subasta ha permitido reducir las comisiones por administración a 0,4I \% del ingreso imponible de los trabajadores hasta julio del 20I8. A partir de agosto, como se ha mencionado, la comisión mínima es de $0,77^{{ }^{17}}$. Para fines exclusivamente ilustrativos, suponemos que el proceso de subasta lleve a que en Colombia al menos una Afp cobre la tarifa mínima de i \% sobre el iвc.

En definitiva, tendremos, entonces, una cotización total de $17 \%$, de los cuales el $\mathrm{I}_{3} \%$ es pagado por los empleadores y el $4 \%$ por los trabajadores. A su vez, el I3 \% de los empleadores se divide en I, $5 \%$ (aproximadamente) para seguros de invalidez y sobrevivencia y i I,5\% que irían al fondo común de vejez de Colpensiones o las cuentas individuales de los trabajadores, según el caso. Por su lado, el $4 \%$ de los trabajadores se reparte entre un I \% (aproximadamente) eliminó la comisión que cobraba por la administración de las pensiones de jubilación. 


\section{Figura 3.}

\section{Distribución de La COTIzación a PENSIONES EN EL CASO de IBC DE DIEZ SALARIOS CON CAMBIOS EN COMISIONES O SEGUROS}

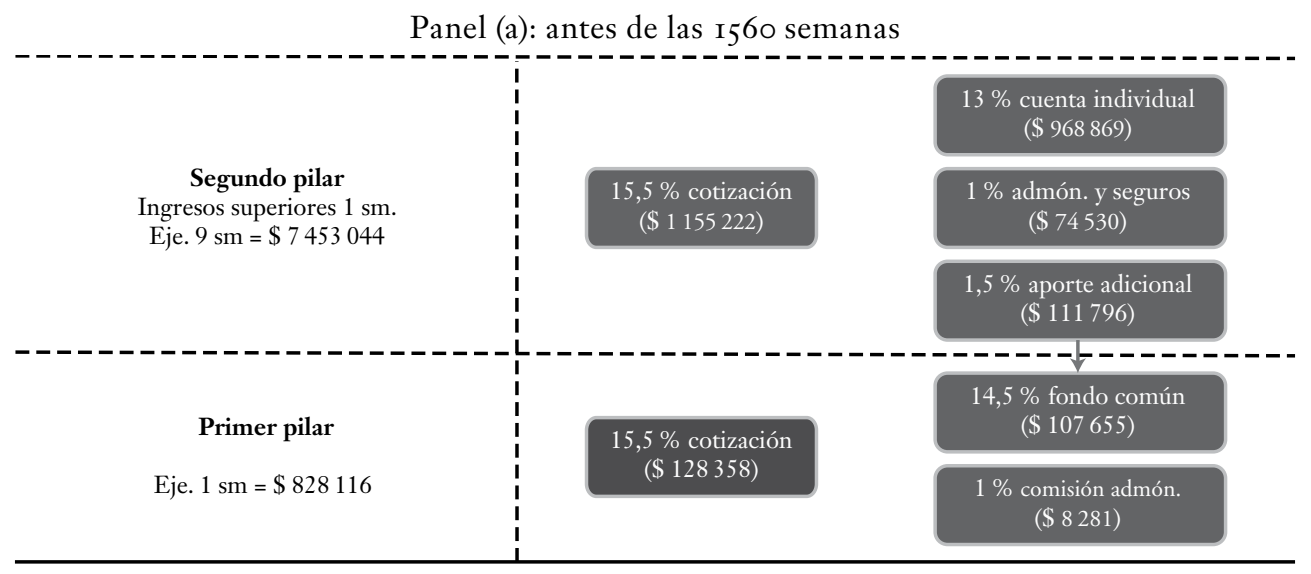

Aportes al FSP de los trabajadores con ingresos mayores a $4 \mathrm{sm}$.

(Colombia Mayor)
$1 \%$ cotización de $10 \mathrm{sm}$ (\$ 82 812)

Panel (b): despues de las 1560 semanas

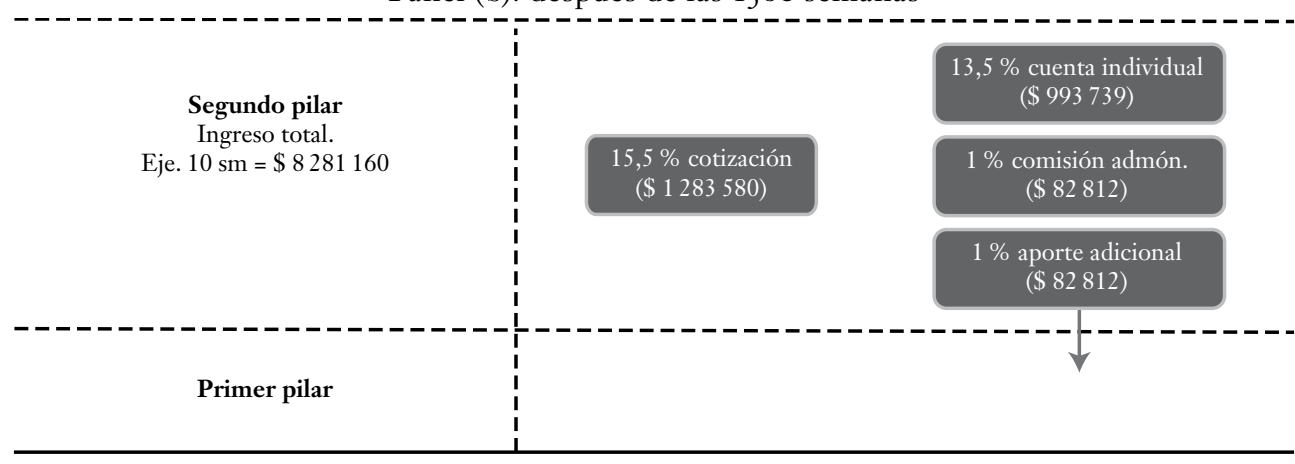

Aportes al FSP de los trabajadores con ingresos mayores a $4 \mathrm{sm}$. (Colombia Mayor)

\section{$1 \%$ Cotización de $10 \mathrm{sm}$} (\$ 82 812)

Fuente: elaboración del autor.

de comisión a las AFP o Colpensiones ${ }^{18}$ y un $3 \%$ que se destinaría al fondo común de vejez de Colpensiones o a las cuentas individuales de los trabajadores, según el caso. La figura 3 resume estos flujos para un trabajador que cotiza con un гвс de diez salarios mínimos antes y después de cotizar I560 semanas.

I 8 En el caso en que el trabajador decida permanecer en una AFP que cobra una comisión de administración mayor, la diferencia será de su responsabilidad, de manera tal que la cotización a su cargo será superior a $4 \%$ y la cotización pensional total será mayor a $17 \%$. 
En ambas figuras solo se muestra un aporte de 15,5\%, recordando que los I, 5 puntos porcentuales de diferencia respecto al $17 \%$ de la cotización total corresponden al pago de los seguros previsionales, efectuado directamente por el empleador a las compañías de seguros.

\section{LOS OTROS CAMBIOS}

Mientras que la reducción de los recargos administrativos y el aumento del IBC máximo a treinta salarios mínimos pueden aplicarse de forma inmediata, el incremento de las semanas a cotizar y de la edad de jubilación deben ser escalonados en el tiempo.

En especial, el aumento de la edad de jubilación de las mujeres deberá ser acompañado por la introducción de créditos pensionales por maternidad ${ }^{\mathrm{T}}$. Por ejemplo, el Estado podría reconocer a las mujeres una suma de dinero por cada hijo nacido vivo, la cual sería depositada en su cuenta personal del segundo pilar o en su cuenta BEP y este depósito no sería sujeto a alguna comisión o aporte de solidaridad.

El incremento del Iвс máximo a treinta salarios mínimos no requeriría de algún cambio legislativo dado que la Ley 797 del 2003 autoriza al Gobierno a subir el Iвс máximo hasta 45 salarios mínimos. Este aumento acentuaría la solidaridad del sistema, ya que, como veremos, para estos niveles de ingresos los aportes que se efectúan superan el capital necesario para obtener una pensión de salario mínimo.

El sistema de pilares propuesto no entrega subsidios en el segundo componente de capitalización y reduce sustancialmente los subsidios unitarios en el componente de prima media. Aproximadamente desde los quince salarios mínimos del IвC los subsidios desaparecen también para los afiliados del primer pilar: los que devengan ingresos más altos aportan más al sistema de lo que reciben como pensión.

Para la gran mayoría de los afiliados, sin embargo, el capital ahorrado en el primer pilar durante su vida productiva seguirá siendo actuarialmente insuficiente para financiar una pensión igual a un salario mínimo. En la medida que aumente la cobertura será entonces urgente encontrar más recursos para la financiación del primer pilar. Tradicionalmente, en Colombia los sistemas de periodos de cotización, montos de dinero que se acreditan al ahorro pensional o en la concesión de mayores mesadas pensionales dependiendo del tipo de esquema pensional considerado, que se conceden a los afiliados que se ven en la imposibilidad de cotizar al sistema de seguridad social por razones socialmente calificadas, por ejemplo, la maternidad, el cuidado de terceros o el desempleo. 
pensiones han sido financiados por contribuciones de trabajadores y empleadores. Es inevitable que en un plazo relativamente cercano habrá que aumentar los aportes de los trabajadores, en la medida en que sus ingresos crezcan y con ello su capacidad de ahorro. Consideramos, sin embargo, que habrá que pensar también en nuevas fórmulas de financiación. En especial de fórmulas que tengan en cuenta las nuevas tendencias en el mercado laboral. Por ejemplo, gravar a las empresas que intensifican el uso del capital en sustitución del trabajo con un aporte sobre el valor de sus activos que iría exclusivamente a financiar el primer pilar. O que parte del descuento del impuesto a la renta al cual tienen derecho las empresas por la compra de activos fijos productivos se destinen a la financiación del primer pilar pensional. Con los mismos fines, se considera conveniente reducir el umbral de Iooo uTv (poco más de 34 millones de pesos) mensuales por encima del cual actualmente se gravan las pensiones a una más razonable cifra de 300 UTv.

En definitiva, más que en un pacto intergeneracional entre jóvenes y ancianos como tradicionalmente se ha concebido, el esquema pensional propuesto se fundamenta en un pacto social sobre la distribución de la riqueza que cuenta el país. La riqueza no la detentan y generan solo los trabajadores activos, sino también el capital y los pensionados.

\section{Conclusiones y comentarios finales}

En las secciones anteriores se ha esbozado la estructura y el funcionamiento de un sistema de pilares que complementa un esquema solidario de prima media con uno de ahorro individual. El sistema propuesto elimina todos los subsidios a las pensiones altas, aunque en su componente de prima media entrega subsidios - decrecientes en valor absoluto- a los afiliados de hasta ingresos medios al concederles una renta vitalicia igual a un salario mínimo.

Vale la pena resaltar que los principales cambios paramétricos propuestos respectan los lineamientos de los Convenios n. ${ }^{\circ}$ IO2 de $195^{2}$ y n. ${ }^{\circ}$ I 28 de 1967 de la Organización Internacional del Trabajo en materia pensional. En especial, el Convenio n..$^{\circ}$ IO2 indica que el "periodo de calificación podrá consistir en treinta años de cotización” y que "la edad prescrita no deberá exceder los sesenta y cinco años". Por su parte el Convenio n. ${ }^{\circ}$ i 28 indica como tasa de reemplazo de la pensión de vejez un porcentaje del $45 \%$, al igual que en el caso de la pensión de sobrevivencia. Además, como observa la misma organización, en un reciente estudio sobre la protección social en América Latina, "la 
modificación o eliminación de la base imponible máxima permitiría mejorar el financiamiento de la seguridad social contributiva" en general ${ }^{20}$.

Este sistema de pilares ofrece incentivos a los afiliados de todo nivel de ingreso a permanecer activos y cotizar aun luego de haber alcanzado la edad o las semanas estatutarias de jubilación. Asimismo, a las personas que por el contrario prefieren anticipar su retiro de la vida activa se les ofrece la posibilidad de hacerlo asumiendo el costo actuarial de su decisión. Junto a la entrega de créditos pensionales por maternidad, las anteriores medidas deberían aumentar la cobertura pensional en el régimen contributivo.

Lo que debe resultar claro, sin embargo, es que en las actuales condiciones sociolaborales aumentar de manera significativa la cobertura en los regímenes pensionales contributivos en Colombia va a ser muy difícil. Entre las personas en edad activas se cuentan unos 750000 trabajadores familiares que, por el hecho de no ser remunerados, tampoco cotizan al RPM o al RAIs. Además, hay 8500 ooo trabajadores por cuenta propia y 600000 jornaleros no afiliados cuyos ingresos laborales promedios alcanzan apenas un $75 \%$ del salario mínimo. Para ellos resulta absolutamente imposible hacerse cargo de los aportes a la seguridad social contributiva.

En este documento no han sido objeto de discusión temas tan importantes y controvertidos como los tiempos de implementación de algunas de las medidas sugeridas - del aumento de la edad de jubilación y de las semanas de cotización, por ejemplo- ni se han avanzado propuestas para cuantificar el costo de comprar semanas de cotización o de anticipar la edad de jubilación. Lo anterior debe ser objeto de estudios actuariales y de la negociación entre actores sociales.

También está por fuera del alcance de este escrito la problemática de la pensión parcial y asistencial o, visto de otra perspectiva, la relación entre el sistema de pilares propuesto y el mecanismo вEP y Colombia Mayor.

En principio, podría pensarse que los afiliados que no logran cumplir con los requisitos para obtener una pensión plena pasarían sus ahorros — sus devoluciones de saldos e indemnizaciones sustitutivas - al mecanismo вер y obtendrían una pensión parcial. Colombia Mayor acogería a todos los colombianos cuyos ingresos fueron tan bajos que en la vida productiva no les permitieron ahorrar lo suficiente para una pensión parcialmente subsidiada y que en la vejez se encuentran en condiciones de pobreza. En ambos sistemas, en la medida que se genere espacio fiscal, aumentar el valor de las mesadas (de los subsidios) será prioritario. Es evidente que en el caso de estas prestaciones 
no, o semi, contributivas existe un doble reto: el de ampliar la cobertura y el de asegurar niveles de suficiencia que garanticen un nivel de seguridad del ingreso.

Por lo mencionado antes acerca de los bajos ingresos laborales de aproximadamente la mitad de la fuerza de trabajo colombiana, se deduce que el mecanismo de los BEP puede convertirse en un instrumento fundamental para que las personas de ingresos inferiores al salario mínimo, y con trabajos inestables, puedan tener alguna protección económica durante su vejez. Habrá que mejorar los estímulos al ahorro voluntario, pero también con respecto a ciertas situaciones laborales habrá que generar condiciones para hacer obligatoria la cotización al mecanismo веP.

En el mediano plazo deberá tomarse en consideración la eliminación del Fondo de Solidaridad Pensional y de los aportes directos que hacen los trabajadores con ingresos superiores a cuatro salarios mínimos. El programa PSAP debería extinguirse y el programa Colombia Mayor debería encontrar financiación directa del presupuesto nacional. Serán necesarios ajustes paramétricos a Colombia Mayor, los principales de los cuales consisten en la elevación de la edad para obtener el beneficio a la edad estatutaria de jubilación y del monto del beneficio a, por lo menos, el valor de la línea de indigencia.

En el mediano plazo deberá también ampliarse el espectro de la cobertura de los créditos pensionales, más allá de los de maternidad aquí propuestos, a situaciones como el cuidado de menores y de ancianos. Así mismo, el mecanismo de créditos por desempleo ya existente en Colombia desde el 2013 deberá ser flexibilizado y mejorado.

Y, finalmente, el esquema de pilares propuesto debe constituir solo el primer paso hacia la introducción de un sistema de protección para la vejez colombiana. En principio, este esquema deberá ser complementado por servicios específicamente dirigidos a los adultos mayores (hasta cierto punto) independientes del tipo de pensión, en especial, por servicios prioritarios como la atención en salud, los alimentos y la vivienda entre otros.

\section{Bibliografía}

Banco Mundial. Envejecimiento sin crisis. Políticas para la protección de los ancianos y la promoción del crecimiento. Washington: Oxford University Press, I994.

Clavijo, Sergio, Nelson Vera, Ekaterina Cuéllar y Andrea Ríos. Elementos para una Reforma Estructural Pensional (REP). Bogotá: ANIF. 20I 7. https://anif.co/sites/default/files/investigaciones/anif-refpensionalo9i 7.pdf

“cut y cтc presentan y socializan sus propuestas para abordar la reforma pensional". Agencia de Información Laboral, 24 de septiembre de 20I8. http://ail.ens.org.co/informe-especial/ cut-y-ctc-presentan-y-socializan-sus-propuestas-para-abordar-la-reforma-pensional/ 
Farné, Stefano y Alejandro Nieto. "Razones para reformar el régimen de ahorro individual". En Cecilia López Montaño y María Claudia Holstine (eds.), La reforma pensional en Colombia. El debate pendiente. Lecciones de América Latina, 56-76. Bogotá: Cisoe y Banco de la República, 2019

Montenegro, Santiago. "El camino es el ahorro. Reforma integral para la vejez". En Cecilia López Montaño y María Claudia Holstine (eds.), La reforma pensional en Colombia. El debate pendiente. Lecciones de América Latina, I28-155. Bogotá: Cisoe y Banco de la República, 20I9.

огт. Presente y futuro de la protección social en América Latina y el Caribe, Panorama Laboral Temático, 4. Lima: ort, Oficina Regional para América Latina y el Caribe, 20I8. https:// www.ilo.org/wcmsp5/groups/public/--americas/_-ro-lima/documents/publication/ wcms_633654.pdf

Orgulloso, Alberto y Héctor Vásquez. "Retos de la política de protección social”. En Cecilia López Montaño y María Claudia Holstine (eds.), La reforma pensional en Colombia. El debate pendiente. Lecciones de América Latina, I76-193. Bogotá: Cisoe y Banco de la República, 2019.

Villar, Leonardo y David Forero. Elementos para una propuesta de reforma del sistema de protección económica para la vejez en Colombia. Cuadernos Fedesarollo 58. Bogotá: Fedesarrollo, 2018. 